\title{
Sonothrombolysis for acute ischemic stroke: a systematic review of randomized controlled trials
}

\author{
Edson Bor-Seng-Shu, M.D., Ph.D., ${ }^{1}$ Ricardo De Carvalho Nogueira, M.D., ${ }^{2}$ \\ Eberval G. Figueiredo, M.D., Ph.D., 1 Eli Faria Evaristo, M.D., Ph.D., 2 \\ Adriana Bastos Conforto, M.D., Ph.D., ${ }^{2,3}$ and Manoel Jacobsen Teixeira, M.D., Ph.D. ${ }^{1}$ \\ Divisions of ${ }^{1}$ Neurological Surgery and ${ }^{2}$ Neurology, Hospital das Clinicas, University of Sao Paulo School of \\ Medicine; and ${ }^{3}$ Instituto Israelita de Ensino e Pesquisa Albert Einstein, Sao Paulo, Brazil
}

\begin{abstract}
Object. Sonothrombolysis has recently been considered an emerging modality for the treatment of stroke. The purpose of the present paper was to review randomized clinical studies concerning the effects of sonothrombolysis associated with tissue plasminogen activator (tPA) on acute ischemic stroke.

Methods. Systematic searches for literature published between January 1996 and July 2011 were performed for studies regarding sonothrombolysis combined with tPA for acute ischemic stroke. Only randomized controlled trials were included. Data extraction was based on ultrasound variables, patient characteristics, and outcome variables (rate of intracranial hemorrhages and arterial recanalization).

Results. Four trials were included in this study; 2 trials evaluated the effect of transcranial Doppler (TCD) ultrasonography on sonothrombolysis, and 2 addressed transcranial color-coded duplex (TCCD) ultrasonography. The frequency of ultrasound waves varied from 1.8 to $2 \mathrm{MHz}$. The duration of thrombus exposure to ultrasound energy ranged from 60 to 120 minutes. Sample sizes were small, recanalization was evaluated at different time points (60 and 120 minutes), and inclusion criteria were heterogeneous. Sonothrombolysis combined with tPA did not lead to an increase in symptomatic intracranial hemorrhagic complications. Two studies demonstrated that patients treated with ultrasound combined with tPA had statistically significant higher rates of recanalization than patients treated with tPA alone.

Conclusions. Despite the heterogeneity and the limitations of the reviewed studies, there is evidence that sonothrombolysis associated with tPA is a safe procedure and results in an increased rate of recanalization in the setting of acute ischemic stroke when wave frequencies and energy intensities of diagnostic ultrasound systems are used. (http://thejns.org/doi/abs/10.3171/2011.10.FOCUS11251)
\end{abstract}

\section{KEY WORDS • sonothrombolysis • stroke • tissue plasminogen activator • transcranial color-coded duplex - transcranial Doppler ultrasonography ultrasound-enhanced thrombolysis}

$\mathrm{S}$ YSTEMIC administration of tPA has been established as an effective therapy for improving neurological outcome after acute ischemic stroke. ${ }^{25}$ Considering the "recanalization hypothesis," which states that the reopening of occluded vessels can save threatened ischemic tissues, faster restoration of cerebral tissue blood flow is associated with better neurological recovery. ${ }^{25}$ Therefore, therapeutic strategies to increase the rate and

\footnotetext{
Abbreviations used in this paper: NIHSS = National Institutes of Health Stroke Scale; TCCD = transcranial color-coded duplex; $\mathrm{TCD}=$ transcranial Doppler; $\mathrm{tPA}=$ tissue plasminogen activator; TRUMBI $=$ Transcranial Low-Frequency Ultrasound-Mediated Thrombolysis in Brain Ischemia.
}

speed of arterial recanalization, without increasing the risk of hemorrhagic complications, are the mainstay of patient treatment and future investigations.

Ultrasound energy has been demonstrated to facilitate activity of fibrinolytic agents, a process known as ultrasound-enhanced thrombolysis, contributing to augmentation of fibrinolysis and arterial recanalization. . $^{1,2,16,21}$ Recently, microbubble sonothrombolysis without tPA has been shown to effectively decrease infarct volumes and occurrence of intracranial hemorrhages in an experimental model of stroke. . $^{11,12}$

The objective of the present study is to review randomized clinical studies regarding ultrasound-enhanced thrombolysis in patients with acute ischemic stroke. 


\section{E. Bor-Seng-Shu et al.}

\section{Methods}

The PubMed database was searched to identify papers published between January 1996 and July 2011 that addressed studies regarding ultrasound-enhanced thrombolysis for acute ischemic stroke in humans. The following search terms were used: "ultrasound-enhanced thrombolysis," "sonothrombolysis," "ultrasound and thrombolysis," "ultrasound and tissue plasminogen activator," "transcranial Doppler and thrombolytic therapy," "transcranial colorcoded duplex and thrombolytic therapy," and "transcranial low-frequency ultrasound and thrombolytic therapy." The reference lists of retrieved articles were also searched. The inclusion criterion was randomized studies on thrombolytic therapy using tPA and ultrasound. Exclusion criteria were as follow: 1) case reports and small case series; 2) nonrandomized studies; 3 ) investigations using intraarterial tPA, those using intraarterial infusion of microspheres using transforaminal insonation for vertebrobasilar circulation, or those using other thrombolytic agents; 4) abstracts of studies presented at international meetings; 5) randomized studies that included patients who had been previously reported on in other articles from the same institution; and 6) studies comprising experimental animal models.

Data extraction was performed based on ultrasound variables (ultrasound technology, frequency of the ultrasound waves, emitted-power output, ultrasound wave mode, and duration of thrombus exposure to ultrasound energy), patient characteristics (sample size, age, neurological status, affected arterial territory, interval from symptom onset to treatment), and outcome variables (rate of asymptomatic and symptomatic intracranial hemorrhagic complications, and of partial and complete arterial recanalization).

Two reviewers (E.B.S.S. and R.C.N.) independently selected the studies and extracted the data; disagreement between the 2 reviewers was resolved by a third independent reviewer (E.G.F).

\section{Results}

There have been 6 randomized controlled trials of sonothrombolysis associated with tPA for acute ischemic stroke. $3,4,15,16,20,23$ Two studies were excluded (one consisted of a pilot study with 3 patients in the control arm, ${ }^{3}$ and the other was published as an abstract $\left.{ }^{20}\right)$. We did not find any randomized study addressing the effects of low-frequency transcranial ultrasound on thrombolysis. Although opinions can diverge (a recent meta-analysis has considered the TRUMBI study as a randomized controlled trial), ${ }^{29}$ we have considered the design of the TRUMBI study as nonrandomized because patients were alternately allocated to standard therapy (tPA alone) and combined therapy (low-frequency ultrasound plus tPA). ${ }^{13}$

\section{Ultrasound Variables Adopted in the Selected Trials}

Two studies evaluated the effects of TCD ultrasonography on thrombolysis, ${ }^{4,22}$ and the remaining two evaluated the TCCD technology. ${ }^{15,16}$ The TCD ultrasonography studies used 2-MHz pulsed ultrasound waves, while TCCD ultrasonography studies used $2-\mathrm{MHz}^{15}$ and
1.8-Mhz pulsed waves ${ }^{16}$ (1 study each). The duration of thrombus exposure to ultrasound energy varied from 60 to 120 minutes. Ultrasound variable data of the studies are shown in Table 1.

\section{Characteristics of the Included Trials}

The TCCD ultrasonography studies were composed of a target group (TCCD ultrasound energy plus tPA) and a control group (only tPA). ${ }^{15,16}$ One TCD ultrasonography study consisted of a target group receiving TCD ultrasound energy plus tPA, and a control group receiving only tPA; ${ }^{2}$ another TCD ultrasonography study had a target group receiving TCD ultrasound energy plus tPA plus microspheres, and a control group receiving only tPA. ${ }^{22}$ Sample sizes, mean ages, median NIHSS scores, affected arterial territories, and the interval between symptom onset and treatment are detailed in Table 2.

\section{Outcome Measures}

Symptomatic intracranial hemorrhages were defined as intracranial hemorrhage appearing on CT scans within 72 hours $^{4,15,16}$ or within 36 hours $^{22}$ from symptom onset associated with neurological deterioration (an increase in NIHSS score of $\geq 4$ points). Arterial recanalization was graded by the TIBI (Thrombolysis in Brain Ischemia) flow criteria (Score 2 or 3, partial recanalization; Score 4 or 5, complete recanalization) ${ }^{4,15,16,22}$ Safety and efficacy outcomes are summarized in Table 3 .

Among the identified studies, the incidence of asymptomatic intracranial hemorrhage ranged from 9\% to $21 \%$ in the sonothrombolysis group and from $0 \%$ to $11 \%$ in the control group. In contrast, symptomatic intracranial hemorrhages occurred in $0 \%-27 \%$ of the cases in the sonothrombolysis group, and in $0 \%-5 \%$ of the patients in the control group. All intracranial hemorrhages were associated with thrombolytic therapy. There were no differences in intracranial hemorrhage rates (asymptomatic and symptomatic hemorrhages) between the sonothrombolysis and control groups of the studies, except for 1 study reporting microsphere dose escalation in which a statistically significant increased rate of symptomatic intracranial hemorrhage was noticed in patients who underwent TCD ultrasonography combined with both TPA and $2.8 \mathrm{ml}$ microspheres; in this subgroup 3 patients died (2 deaths were attributed to symptomatic intracranial hemorrhage)..$^{22}$ Concerning a TCCD ultrasonography study, ${ }^{16}$ 3 patients $(15.8 \%)$ from the sonothrombolysis group and 2 patients (11.8\%) from the control group died of spaceoccupying infarction and symptomatic intracranial hemorrhage. Craniotomy was performed in 1 patient in the sonothrombolysis group due to space-occupying infarction and symptomatic intracranial hemorrhage.

Complete arterial recanalization rates varied from $15 \%$ to $67 \%$ in the sonothrombolysis group and from $11 \%$ to $33 \%$ in the control group. Two studies, one using TCD ultrasonography and the other using TCCD ultrasonography, ${ }^{4,16}$ demonstrated that patients treated with ultrasound energy in association with tPA had statistically significantly higher rates of both complete recanalization and neurological improvement than those treated with tPA only. In a TCD ultrasonography study, ${ }^{4}$ complete recana- 


\section{Ultrasound-enhanced thrombolysis}

TABLE 1: Ultrasound variables in randomized studies of sonothrombolysis*

\begin{tabular}{lccccc}
\hline \multicolumn{1}{c}{ Authors \& Year } & $\begin{array}{c}\text { Ultrasound } \\
\text { Technology }\end{array}$ & Frequency (MHz) & Emitted-Power Output (mW/cm²) & Mode & $\begin{array}{c}\text { Duration of Insonation } \\
\text { (mins) }\end{array}$ \\
\hline Eggers et al., 2003 & TCCD & $2-4$ & 179 & pulsed & 60 \\
Alexandrov et al., 2004 & TCD & 2 & $<750$ & pulsed & 120 \\
Eggers et al., 2008 & TCCD & 1.8 & 179 & pulsed & 60 \\
Molina et al., 2009 & TCD & 2 & NR & pulsed & 90 \\
\hline
\end{tabular}

${ }^{*} \mathrm{NR}=$ not reported.

lization or significant clinical recovery within 2 hours after intravenous tPA bolus occurred in $49 \%$ of patients from the sonothrombolysis group, compared with $30 \%$ of patients from the control group $(\mathrm{p}=0.03)$. In a TCCD ultrasonography study, ${ }^{16}$ complete or partial recanalization after 1 hour was found in $57.9 \%$ of the sonothrombolysis group and $22.2 \%$ of the control group $(\mathrm{p}=0.045)$; after 3 months, Barthel Index greater than 95 occurred in 8 patients of the sonothrombolysis group (none from the control group) $(\mathrm{p}=0.003)$.

\section{Discussion}

Only 4 studies met inclusion criteria of this systematic review. 4,15,16,22 Two studies evaluated the role of TCD ultrasonography on sonothrombolysis ${ }^{4,22}$ and 2 evaluated the role of TCCD technology. ${ }^{15,16}$ The frequency of ultrasound waves varied from 1.8 to $2 \mathrm{MHz}$. The duration of thrombus exposure to ultrasound energy ranged from 60 to 120 minutes. Sample sizes were small. Recanalization was evaluated at different time points (60 and 120 minutes). Inclusion criteria were heterogeneous; 2 TCCD studies included only patients with proximal middle cerebral artery occlusion without residual flow, a condition in which recanalization is more difficult; ${ }^{15,16} 1$ TCD study had a target group receiving ultrasound energy, intravenous tPA, and microspheres. ${ }^{22}$ Therefore, comparisons among the studies are challenging, and conclusions are difficult to draw. Despite the heterogeneity of the included studies, ultrasound-enhanced thrombolysis seems to be a safe procedure and is associated with a significantly higher rate of arterial recanalization in the acute ischemic stroke setting. It is worth noting that this conclusion was reached considering results of 2 randomized controlled trials (one assessing TCD and the other TCCD).,16
Issues related to instrumentation in ultrasonography are of fundamental importance to interpret the studies regarding sonothrombolysis in acute ischemic stroke. The following 3 different ultrasound modalities were used to increase the thrombolytic activity of tPA: TCD ultrasonography, TCCD ultrasonography, and therapeutic transcranial low-frequency ultrasonography. ${ }^{27,28}$ These devices generate ultrasound beams that differ greatly in acoustic properties, such as frequency, mechanical index, and the amount of brain tissue included in the beam (that is, areas of the brain exposed to ultrasound energy). Transcranial Doppler ultrasonography equipped with a $2-\mathrm{MHz}$ transducer has been widely used for evaluating patients with acute ischemic stroke, ${ }^{27,28}$ traumatic brain injury,,${ }^{9,10}$ and aneurysmal subarachnoid hemorrhage. ${ }^{7}$ This nonimaging and hand-held ultrasound examination device provides real-time blood flow velocity from cerebral arteries that can indicate arterial occlusion or recanalization, embolization, functional status of collateral circulatory pathways, and blood steal phenomenon. ${ }^{27,28}$ For diagnostic purposes, the emitted-power output is usually set at the maximal achievable level below the allowed limit of $720 \mathrm{~mW}$ with selected insonation deaths, and the sample volumes, or gates of insonation, are set at 3-6 $\mathrm{mm}$ for power motion Doppler devices and $10-15 \mathrm{~mm}$ for other single channel devices. ${ }^{27,28}$

While using TCD ultrasonography to monitor tPA thrombolysis, Alexandrov et al. ${ }^{2}$ incidentally suspected the ability of TCD ultrasonography to facilitate arterial recanalization in patients with acute ischemic stroke treated with intravenous tPA, a phenomenon confirmed afterward by the CLOTBUST (Combined Lysis of Thrombus in Brain ischemia using transcranial Ultrasound and Systemic tPA) trial. When signs of arterial occlusion are detected, the TCD ultrasound beam can be continu-

TABLE 2: Characteristics of the patients in randomized clinical trials of sonothrombolysis*

\begin{tabular}{|c|c|c|c|c|c|c|}
\hline Authors \& Year & Target Group† & No. of Cases (target vs control) & $\begin{array}{l}\text { Mean Age } \\
\text { (yrs) } \ddagger\end{array}$ & $\begin{array}{c}\text { Median NIHSS } \\
\text { Score (range) }\end{array}$ & Occluded Artery & $\begin{array}{c}\text { Symptom Onset to } \\
\text { Treatment (hrs) }\end{array}$ \\
\hline Eggers et al., 2003 & $\mathrm{TCCD}+\mathrm{tPA}$ & 11 vs 14 & $61 \pm 9$ & $18(9-25)$ & $\operatorname{MCA}\left(M_{1}\right)$ & $\leq 3$ \\
\hline Alexandrov et al., 2004 & $T C D+t P A$ & 63 vs 63 & $69 \pm 13$ & $16(4-34)$ & MCA & $\leq 3$ \\
\hline Eggers et al., 2008 & $\mathrm{TCCD}+\mathrm{tPA}$ & 19 vs 18 & $61 \pm 10$ & $17.5(12-23)$ & $\operatorname{MCA}\left(M_{1}\right)$ & $\leq 3$ \\
\hline Molina et al., 2009 & $\mathrm{TCD}+\mathrm{tPA}+\mu \mathrm{S}$ & $12(1.4 \mathrm{ml} \mu \mathrm{S}), 11(2.8 \mathrm{ml} \mu \mathrm{S})$ vs 12 & $65 \pm 14$ & $12(4-21)$ & MCA, PCA & $\leq 3$ \\
\hline
\end{tabular}

* $\mu \mathrm{S}=$ microspheres; $\mathrm{MCA}=$ middle cerebral artery; $\mathrm{PCA}=$ posterior cerebral artery.

$\dagger$ In all studies, the control group received only tPA.

$\ddagger$ Values are presented as means \pm SDs. 


\section{E. Bor-Seng-Shu et al.}

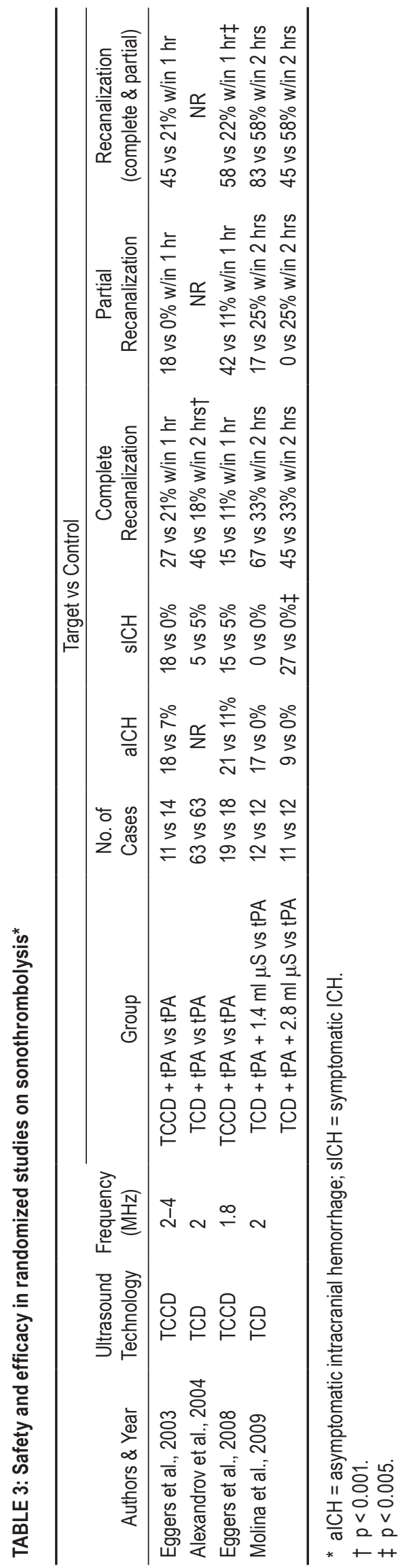

ously focused at the presumed thrombus location, allowing both sonothrombolysis and continuous monitoring of the recanalization process. Unfortunately, this method is highly dependent on the skill of the TCD ultrasonography operator. It is unrealistic to expect that an unskilled TCD ultrasonography clinician can develop skills for a rapid and accurate location of cerebral arterial occlusions., ${ }^{4,27,28}$

Transcranial color-coded duplex is another ultrasound method that, like TCD ultrasonography, provides real-time blood flow dynamics of cerebral arteries. However, unlike TCD technology, TCCD transducers generate multiple small ultrasound beams at dual emitting frequencies, one for gray scale imaging and another for Doppler imaging; hence, besides assessment of cerebral hemodynamics, this technology can also afford both arterial location on color flow imaging and imaging of the brain on B-mode ultrasonography. 5,8,27,28 The TCCD ultrasound beam includes a larger brain area (a greater amount of brain tissue is exposed to ultrasound energy) compared with the more focal TCD ultrasound beam. Among the limitations of TCCD ultrasonography, there are no head frames for transducer fixation (recanalization monitoring is carried out using handheld probes), the mechanical index (an indicator of the likelihood of mechanical biological effects, that is, streaming and cavitation) of the TCCD ultrasonography is higher than that for TCD ultrasonography, and no dose escalation study has been performed to determine the levels of ultrasound intensity needed to enhance thrombolysis without risks. ${ }^{27,28}$

Therapeutic transcranial low-frequency ultrasonography is a nonimaging and nondiagnostic ultrasound technology equipped with a transducer composed of 4 elements arranged in a diamond pattern. This technology uses unfocused, low-frequency ultrasound $(300 \pm 1.5 \mathrm{kHz}$ to avoid standing waves), a temporal average spatial peak intensity of $700 \mathrm{~mW} / \mathrm{cm}^{2}$, an average temporal pressure less than 1 atmosphere $(<101 \mathrm{kPa}$ for avoiding cavitation), a mechanical index less than 0.2 , a thermal index in soft tissue less than 0.5 , and a cranial thermal index of approximately 4 . The higher cranial thermal index was addressed through the use of a cooling pad and a thermal sensor to detect excessive heating. To decrease the thermal effects, ultrasound is emitted in a pulsed fashion with a $5 \%$ duty cycle and a pulse repetition frequency of $100 \mathrm{~Hz}$ (cycle/pulse ratio of 225). Despite leading to a higher rate of recanalization, this method sonicates the vessels and brain nonspecifically. The TRUMBI trial was stopped because intracranial hemorrhages were frequently found, even in areas unaffected by ischemia. ${ }^{13,27,28}$ The development of high-intensity, focused ultrasound with MR imaging guidance may minimize hemorrhagic complications in the future.

A recent comprehensive review and meta-analysis has assessed the safety and efficacy of ultrasound-enhanced tPA thrombolysis in the treatment of patients with acute ischemic stroke. ${ }^{29}$ Taking into account 6 randomized (comprising 224 patients) and 3 nonrandomized (comprising 192 patients) studies, the authors concluded that sonothrombolysis using TCD or TCCD ultrasonography appears to be safe and leads to higher rates of complete recanalization when compared with intravenous tPA thrombolysis. 
Sonothrombolysis using TCD or TCCD ultrasonography, with or without microspheres, is associated with a nearly 3 -fold increased likelihood of complete recanalization and an approximately 2-fold higher likelihood of functional independence at 3 months. We stressed that this meta-analysis included randomized and nonrandomized studies, as well as abstracts of studies presented at international meetings, and focused on quantitative analysis of them, without taking into consideration ultrasound variables such as type of ultrasound technology, frequency of ultrasound waves, emitted power output, ultrasound wave mode, and duration of thrombus exposure to ultrasound energy, while our manuscript focused on qualitative analysis of Class I evidence studies, classifying them in terms of ultrasound variables, presence of microspheres, sample characteristics, and outcome measures. Although both reviews have provided similar findings (that is, ultrasound-enhanced thrombolysis seems to be a safe procedure and is associated with a significantly higher rate of arterial recanalization in acute ischemic stroke), our study revealed that only 2 randomized controlled studies, one using TCD and the other using TCCD, support these conclusions.

Ultrasound energy has been experimentally demonstrated to improve the thrombolytic effects of tPA. ${ }^{6}$ The mechanisms of action are still under debate; however, there is evidence of at least 4 contributing effects as follows: 1) rectified diffusion, which provides a pumping effect to transport drugs into the thrombus; 2) reformation and opening of the fibrin matrix under ultrasound exposure, which enhances drug diffusion; 3) cleaving of fibrin polymers to extend the surface for thrombolytic interaction; and 4) improvement of binding of recombinant tPA to fibrin. ${ }^{14}$ Concerning the frequency range used for diagnostic ultrasound examinations, the mechanical pressure wave of the ultrasound energy propagates through the tissues, induces fluid motion, and helps tPA to reach the binding sites..$^{17}$ In addition, $2-\mathrm{MHz}$ ultrasound waves enhance tPA-thrombus dissolution by fluid streaming around the clot surface and disaggregation of fibrin fibers, which results in a greater amount of binding sites for tPA without heating or cavitation. ${ }^{3}$ In stroke patients treated with tPA, intravenous infusion of microbubbles or microspheres has been proved to accelerate clot lysis during 2-MHz ultrasound monitoring, by transmitting energy momentum from an ultrasound wave to residual flow. ${ }^{3,11,12,22,23}$ These mechanisms support the findings of sonothrombolysis in the clinical setting.

\section{Conclusions}

Despite the limitations of the included studies, there is evidence that sonothrombolysis associated with tPA with frequencies and energy intensities in a range of diagnostic ultrasound devices is safe and results in an increased rate of arterial recanalization in acute ischemic stroke. Future studies should focus on the development of the following: 1) microspheres associated with ultrasound for augmenting brain perfusion and drug delivery within the penumbra area; ${ }^{18}$ 2) an operator-independent ultrasound device that can be used by medical personnel irrespective of experience with TCD or TCCD ultra- sonography examinations; ${ }^{27,28} 3$ ) intraarterial ultrasound devices for thrombolysis; ${ }^{26}$ and 4) ultrasound catheters for minimally invasive evacuation of intracranial hematomas as a complication of thrombolytic therapy or other causes..$^{21,24}$ In the near future, it is possible that high-intensity focused ultrasound neurosurgery for brain tumors, Parkinson disease, epilepsy, and other conditions may become a reality. ${ }^{19}$

\section{Disclosure}

The authors report no conflict of interest concerning the materials or methods used in this study or the findings specified in this paper.

Author contributions to the study and manuscript preparation include the following. Conception and design: Bor-Seng-Shu, Nogueira, Figueiredo. Acquisition of data: Bor-Seng-Shu, Nogueira, Figueiredo. Analysis and interpretation of data: all authors. Drafting the article: Bor-Seng-Shu, Nogueira. Critically revising the article: all authors. Reviewed submitted version of manuscript: all authors. Approved the final version of the manuscript on behalf of all authors: Bor-Seng-Shu. Administrative/technical/material support: Teixeira. Study supervision: Teixeira.

\section{References}

1. Alexandrov AV: Ultrasound enhanced thrombolysis for stroke. Int J Stroke 1:26-29, 2006

2. Alexandrov AV, Demchuk AM, Felberg RA, Christou I, Barber PA, Burgin WS, et al: High rate of complete recanalization and dramatic clinical recovery during tPA infusion when continuously monitored with $2-\mathrm{MHz}$ transcranial doppler monitoring. Stroke 31:610-614, 2000

3. Alexandrov AV, Mikulik R, Ribo M, Sharma VK, Lao AY, Tsivgoulis G, et al: A pilot randomized clinical safety study of sonothrombolysis augmentation with ultrasound-activated perflutren-lipid microspheres for acute ischemic stroke. Stroke 39:1464-1469, 2008

4. Alexandrov AV, Molina CA, Grotta JC, Garami Z, Ford SR, Alvarez-Sabin J, et al: Ultrasound-enhanced systemic thrombolysis for acute ischemic stroke. N Engl J Med 351:21702178, 2004

5. Barsottini OG, Felício AC, de Carvalho Aguiar P, GodeiroJunior C, Pedroso JL, de Aquino CC, et al: Heterozygous exon 3 deletion in the Parkin gene in a patient with clinical and radiological MSA-C phenotype. Clin Neurol Neurosurg 113: 404-406, 2011

6. Blinc A, Francis CW, Trudnowski JL, Carstensen EL: Characterization of ultrasound-potentiated fibrinolysis in vitro. Blood 81:2636-2643, 1993

7. Bor-Seng-Shu E, de-Lima-Oliveira M, Teixeira MJ, Panerai RB: Predicting symptomatic cerebral vasospasm after aneurysmal subarachnoid hemorrhage. Neurosurgery 69:E501E502, 2011 (Letter)

8. Bor-Seng-Shu E, Fonoff ET, Barbosa ER, Teixeira MJ: Substantia nigra hyperechogenicity in Parkinson's disease. Acta Neurochir (Wien) 152:2085-2087, 2010 (Letter)

9. Bor-Seng-Shu E, Hirsch R, Teixeira MJ, De Andrade AF, Marino R Jr: Cerebral hemodynamic changes gauged by transcranial Doppler ultrasonography in patients with posttraumatic brain swelling treated by surgical decompression. J Neurosurg 104:93-100, 2006

10. Bor-Seng-Shu E, Teixeira MJ, Hirsch R, Andrade AF, Marino R Jr: Transcranial doppler sonography in two patients who underwent decompressive craniectomy for traumatic brain swelling: report of two cases. Arq Neuropsiquiatr 62 (3A): 715-721, 2004

11. Culp WC, Flores R, Brown AT, Lowery JD, Roberson PK, 


\section{E. Bor-Seng-Shu et al.}

Hennings LJ, et al: Successful microbubble sonothrombolysis without tissue-type plasminogen activator in a rabbit model of acute ischemic stroke. Stroke 42:2280-2285, 2011

12. Culp WC, Porter TR, Lowery J, Xie F, Roberson PK, Marky $\mathrm{L}$ : Intracranial clot lysis with intravenous microbubbles and transcranial ultrasound in swine. Stroke 35:2407-2411, 2004

13. Daffertshofer M, Gass A, Ringleb P, Sitzer M, Sliwka U, Els T, et al: Transcranial low-frequency ultrasound-mediated thrombolysis in brain ischemia: increased risk of hemorrhage with combined ultrasound and tissue plasminogen activator: results of a phase II clinical trial. Stroke 36:1441-1446, 2005

14. Daffertshofer M, Hennerici M: Ultrasound in the treatment of ischaemic stroke. Lancet Neurol 2:283-290, 2003

15. Eggers J, Koch B, Meyer K, König I, Seidel G: Effect of ultrasound on thrombolysis of middle cerebral artery occlusion. Ann Neurol 53:797-800, 2003

16. Eggers J, König IR, Koch B, Händler G, Seidel G: Sonothrombolysis with transcranial color-coded sonography and recombinant tissue-type plasminogen activator in acute middle cerebral artery main stem occlusion: results from a randomized study. Stroke 39:1470-1475, 2008

17. Francis CW, Blinc A, Lee S, Cox C: Ultrasound accelerates transport of recombinant tissue plasminogen activator into clots. Ultrasound Med Biol 21:419-424, 1995

18. Holland CK, McPherson DD: Echogenic lipsomes for targeted drug delivery. Proc IEEE Int Symp Biomed Imaging 2009:755-758, 2009

19. Jagannathan J, Sanghvi NT, Crum LA, Yen CP, Medel R, Dumont AS, et al: High-intensity focused ultrasound surgery of the brain: part 1-A historical perspective with modern applications. Neurosurgery 64:201-211, 2009

20. Larrue V, Viguier A, Arnaud C, Cognard C, Petit R, Rigal $\mathrm{M}$, et al: Transcranial ultrasound combined with intravenous microbubbles and tissue plasminogen activator for acute ischemic stroke: a randomized controlled study. Stroke 38:472, 2007

21. Medel R, Crowley RW, McKisic MS, Dumont AS, Kassell NF: Sonothrombolysis: an emerging modality for the management of stroke. Neurosurgery 65:979-993, 2009
22. Molina CA, Barreto AD, Tsivgoulis G, Sierzenski P, Malkoff MD, Rubiera M, et al: Transcranial ultrasound in clinical sonothrombolysis (TUCSON) trial. Ann Neurol 66:28-38, 2009

23. Molina CA, Ribo M, Rubiera M, Montaner J, Santamarina E, Delgado-Mederos R, et al: Microbubble administration accelerates clot lysis during continuous $2-\mathrm{MHz}$ ultrasound monitoring in stroke patients treated with intravenous tissue plasminogen activator. Stroke 37:425-429, 2006

24. Newell DW, Shah MM, Wilcox R, Hansmann DR, Melnychuk E, Muschelli J, et al: Minimally invasive evacuation of spontaneous intracerebral hemorrhage using sonothrombolysis. Clinical article. J Neurosurg 115:592-601, 2011

25. Rha JH, Saver JL: The impact of recanalization on ischemic stroke outcome: a meta-analysis. Stroke 38:967-973, 2007

26. Soltani A, Singhal R, Obtera M, Roy RA, Clark WM, Hansmann DR: Potentiating intra-arterial sonothrombolysis for acute ischemic stroke by the addition of the ultrasound contrast agents $\left(\mathrm{Optison}^{\mathrm{TM}} \&\right.$ SonoVue $\left.{ }^{\circledR}\right)$. J Thromb Thrombolysis 31:71-84, 2011

27. Tsivgoulis G, Alexandrov AV: Ultrasound-enhanced thrombolysis in acute ischemic stroke: potential, failures, and safety. Neurotherapeutics 4:420-427, 2007

28. Tsivgoulis G, Culp WC, Alexandrov AV: Ultrasound enhanced thrombolysis in acute arterial ischemia. Ultrasonics 48:303311,2008

29. Tsivgoulis G, Eggers J, Ribo M, Perren F, Saqqur M, Rubiera M, et al: Safety and efficacy of ultrasound-enhanced thrombolysis: a comprehensive review and meta-analysis of randomized and nonrandomized studies. Stroke 41:280-287, 2010

Manuscript submitted September 15, 2011

Accepted October 31, 2011.

Please include this information when citing this paper: DOI: 10.3171/2011.10.FOCUS11251

Address correspondence to: Edson Bor-Seng-Shu, M.D., Ph.D., Rua Loefgreen, 1272, CEP 04040-001, Sao Paulo, SP, Brazil. email: edsonshu@hotmail.com. 